\title{
Design of Galfenol and Alfenol microstructures for bending mode energy harvesters
}

\author{
Şafak Çallıoğlu ${ }^{1,2}$ and Pınar Acar ${ }^{2, *}$ (i) \\ ${ }^{1}$ Student at Bilkent University, Ankara, Turkey \\ ${ }^{2}$ Virginia Polytechnic Institute and State University, Department of Mechanical Engineering, 445 Goodwin Hall, 635 Prices Fork \\ Road, Blacksburg, VA 24061, USA
}

Received: 17 March 2020

Accepted: 13 May 2020

Published online:

8 June 2020

(C) Springer Science+Business Media, LLC, part of Springer Nature 2020

\begin{abstract}
The present work addresses the microstructural design of Galfenol and Alfenol, which are magnetostrictive materials used in bending mode energy harvesters. Galfenol and Alfenol have been attracting much interest during the last few decades as they provide bi-directional coupling between mechanical and magnetic properties.This magneto-mechanical coupling generates electrical energy in distinctive energy harvesting mechanisms that use mechanical or magnetic input. One favorable approach would be enhancing the energy efficiency by optimizing the material properties through the control and design of the underlying microstructure. However, to the best of our knowledge, there is no effort for designing Galfenol and Alfenol microstructures to maximize energy efficiency. Therefore, we present a computational design study to find the optimum Galfenol and Alfenol microstructures that can maximize the energy efficiency for bending mode energy harvesters. We model the effects of the crystallographic texture on the mechanical and magnetic properties and develop a computational model to represent the magneto-mechanical coupling.The outcomes of our computational strategy revealed that the optimum design solutions are highly textured polycrystals. Moreover, the optimum Galfenol and Alfenol microstructures are found to provide around $80 \%$ and 32\% energy efficiency, which are higher than the known optimum efficiency level for the same materials.
\end{abstract}

\section{Introduction}

Magnetostrictive materials are the class of multiphysics materials that are used extensively in many different areas. They are essentially known to experience mechanical deformation under an applied magnetic field. The metallic (polycrystalline) magnetostrictive materials exhibit directional material properties as they have preferred crystalline orientations that provide higher magnetic moments compared to other microstructural orientations. This can be explained with electrical interactions between the

Address correspondence to E-mail: pacar@vt.edu 
attached electronic charge clouds and the neighboring charged ions [1]. Therefore, shift and rotation of boundaries between magnetic domains emerge when the magnetostrictive materials are exposed to the magnetic field, as magnetic domains in the material align with the applied magnetic field. This feature causes a mechanical deformation. Similarly, the mechanical input applied to magnetostrictive materials can produce the magnetic field that can be converted into electrical output.

One of the promising magnetostrictive materials is Alfenol, which has been known since the 1940s [2]. Alfenol has a body-centered cubic (BCC) crystal system. It demonstrates a considerable magnetoelastic coupling [3] and high Curie temperature [4]. The applications of Alfenol include transducers [5] and sensors [6-8].

A more recent polycrystalline magnetostrictive material is Galfenol developed by Clark et al. in the 2000s [9]. It demonstrates a BCC crystal system. Galfenol was shown to combine significant magnetostriction and strong mechanical properties [9] with the capability of being folded into various shapes [10]. Moreover, its properties are less dependent on the temperature changes $[9,11,12]$, and thus, it can operate in a wide temperature range compared to other magnetostrictive materials. All these outstanding properties have qualified Galfenol as a desirable material in different applications, such as transducers [13-15], MEMS [16], actuators [17-19] and other sensors [19-23].

Advancements in technology stimulate to build energy harvesting devices that can extract electrical energy from the environment as a supplement to used batteries or alternative of conventional power supplies. One class of the energy harvesters is the magnetostrictive energy harvester, which is currently used in different areas such as ocean wave generators [24, 25], down-hole drills [26], vehicle tires [27] and rotating shaft [28]. Many experimental studies have been conducted for manufacturing these magnetostrictive energy harvesters in the axial mode for Galfenol [29, 30], as well as in bending mode for Galfenol [31-34] and for Alfenol [35, 36].

Materials-by-design has recently become an emerging research field since the introduction of the Integrated Computational Materials Engineering (ICME) [37] paradigm. It focuses on the improvement of the material properties by optimizing the material microstructure. The microstructural texture can be controlled during deformation processing so that the processing route can be designed to obtain the desired microstructures that produce targeted material properties. This was accomplished before the graphical quantification of property-performance relations using the property cross-plots, as presented by Ashby [38]. With the recent developments in materials-by-design, a more systematic design approach was found to combine the processing, structure and property through computational material models [39]. Similarly, in this work, we focus on controlling the microstructural texture of Alfenol and Galfenol to achieve desired material properties that maximize energy efficiency when a mechanical input is received in the bending mode. The microstructure design was previously studied by our group to optimize the vibration frequencies [40] and magnetostrictive strain [41, 42] of Galfenol. However, these studies [40-42] addressed the optimization of different volume-averaged material properties and did not focus on the magneto-mechanical coupling. To the best of our knowledge, there is no effort in the literature to optimize the Alfenol and Galfenol microstructures to maximize energy efficiency by developing a computational model for the magnetomechanical coupling. Therefore, in this work, we investigate the microstructure design of the magnetostrictive materials, Galfenol and Alfenol, using computational methods and consider the bending mode electric power harvester, which is based on Haynes, Yoo and Flatau's paper [43].

\section{Microstructure modeling}

The microstructural texture is represented with the orientation distribution function (ODF), denoted by $A$. The ODF represents the local densities of crystals over the crystallographic orientation space. The ODF is a measure of the microstructural orientations. To describe the finite number of crystallographic orientations in the microstructure, the ODF is discretized using a finite element (FE) approach in the Rodrigues orientation space. The details on the FE scheme and Rodrigues parameterization can be found in our earlier studies [40,41,44-46]. Using this scheme, a volume normalization constraint on the ODFs is defined as follows: 


$$
\begin{aligned}
& \oint_{R} A \mathrm{~d} v=1 \\
& \Rightarrow \sum_{n=1}^{N_{\text {element }}} \sum_{m=1}^{N_{\text {int }}} A\left(r_{m}\right) w_{m}\left|J_{n}\right| \frac{1}{\left(1+r_{m} \cdot r_{m}\right)^{2}}=1
\end{aligned}
$$

where $N_{\text {element }}$ and $N_{\text {int }}$ represent the number of FEs and integration points, respectively. $A\left(r_{m}\right)$ is the volume fraction value at $m$ th integration point with $r_{m}$ parameter, $w_{m}$ is integration weight associated with mth integration point, $\left|J_{n}\right|$ is Jacobian determinant of $n$th element. Equation (1) is equivalent to a linear expression in the ODF:

$q^{T} A=1$

where $q$ shows the volume normalization vector. Similarly, the volume-averaged property can be computed with the expression given next:

$$
\begin{aligned}
\langle\chi\rangle & =\oint_{R} \chi(r) A(r, t) \mathrm{d} v \\
& =\sum_{n=1}^{N_{\text {elem }}} \sum_{m=1}^{N_{\text {int }}} \chi\left(r_{m}\right) A\left(r_{m}\right) w_{m}\left|J_{n}\right| \frac{1}{\left(1+r_{m} \cdot r_{m}\right)^{2}}
\end{aligned}
$$

where the ODF, $A \geq 0$, is a function of orientation $r$, and time $t$ (during processing).

The volume-averaging equation can be written in following the linear form:

$$
\langle\chi\rangle=p^{T} A
$$

In this work, the modeling of the magneto-mechanical coupling requires the computation of Young's modulus in the direction of the applied load $\left(E_{11}\right)$. It is inversely related to the compliance, $S$, that is also the inverse of the volume-averaged stiffness, $C$. The computation procedure for Young's modulus is summarized below:

$$
\begin{aligned}
& C=p^{T} A \\
& S=C^{-1} \\
& E_{11}=\frac{1}{S(1,1)}
\end{aligned}
$$

\section{Methodology}

\section{Magnetostrictive strain calculation}

The magnetostrictive strain is previously defined by the following equation [47].

$$
\lambda=\left[\begin{array}{ccc}
\lambda_{100}\left(m_{x}^{2}-1 / 3\right) & \lambda_{111}\left(m_{x} m_{y}\right) & \lambda_{111}\left(m_{x} m_{z}\right) \\
\lambda_{111}\left(m_{y} m_{x}\right) & \lambda_{100}\left(m_{y}^{2}-1 / 3\right) & \lambda_{111}\left(m_{y} m_{z}\right) \\
\lambda_{111}\left(m_{z} m_{x}\right) & \lambda_{111}\left(m_{z} m_{y}\right) & \lambda_{100}\left(m_{z}^{2}-1 / 3\right)
\end{array}\right]
$$

where $\lambda_{100}$ and $\lambda_{111}$ characterizes the change in normal strain along $\langle 100\rangle$ and $\langle 111\rangle$ direction, respectively, $m_{x}, m_{y}, m_{z}$ are the components of unit vector $m$ indicating the magnetization direction of a single crystal.

The magnetostrictive materials develop 3 forms of energy: (1) internal energy, (2) magnetic energy, (3) interaction energy. As explained in the introduction, the magnetic domains of the magnetostrictive materials rotate due to the applied magnetic field, which produces internal energy as described next [47]:

$$
E_{I}=K_{1}\left(m_{x}^{2} m_{y}^{2}+m_{x}^{2} m_{z}^{2}+m_{y}^{2} m_{z}^{2}\right)+K_{2}\left(m_{x}^{2} m_{y}^{2} m_{z}^{2}\right)
$$

The magnetic energy created by the applied magnetic field is given as follows:

$E_{H}=-\mu_{0} M H(m \cdot n)$

where $n$ is the unit vector representing the direction of the applied magnetic field, and $M$ is the magnetization of the domain.

The interaction energy between the applied and magnetostrictive strain is defined as:

$E_{\sigma}=-(\sigma \cdot \lambda)$

At ideal conditions, it is expected that all magnetic domains are aligned in such a way that minimal energy is satisfied. However, the domain magnetization obeys a Boltzmann-like distribution, caused by the increase in entropy due to non-ideal conditions. The probability of having the magnetization direction, $m$, can be expressed using the given exponential form of energy summation [47].

$P(m)=\alpha \mathrm{e}^{\frac{-\left(E_{I}+E_{H}+E_{\sigma}\right)}{\Omega}}$

where $\alpha$ is a constant, $\Omega$ is a parameter representing the spread of the magnetization direction from the ideal direction that provides minimal energy.

Therefore, the average magnetostrictive strain can be found with the following equation [47]:

$\lambda_{s}=\frac{\int P(m) R^{T} \lambda R \mathrm{~d} m}{\int P(m) \mathrm{d} m}$

where $\lambda_{s}$ is the average magnetostrictive strain, $R$ is the rotation tensor, $P(m)$ is the probability of having 
the magnetization direction, $m$. The magnetic domains $(m)$ describe the anisotropy of the magnetostrictive strain. The magnetostrictive strain, $\lambda_{s}$, values along the $\langle 100\rangle$ direction are visualized for Galfenol and Alfenol in the Rodrigues orientation space in Figs. 1 and 2, respectively. The directions are also shown in terms of the pole figures in the $\langle 111\rangle$, $\langle 100\rangle$, and $\langle 110\rangle$ directions in Figs. 3 and 4 for Galfenol and Alfenol, respectively.

It can be interpreted from Figs. 1, 2, 3 and 4 that the microstructural orientations providing higher magnetostrictive strain values for Galfenol and Alfenol are different.

\section{Computation of piezomagnetic coupling coefficient}

Many material models can be constructed using linear piezomagnetic equations including the coupling parameter, $d$. The coupling parameter is essentially defined as the ratio of the average magnetostrictive strain, $\lambda_{s}$, and the applied magnetic field, $H$ [48]:

$d=\frac{\lambda_{s}}{H}$

The $\lambda_{s}$ and $H$ are in both $\langle 100\rangle$ direction.

\section{Magnetomechanical modeling}

The system that we analyze consists of a magnetostrictive rod with a tip mass, permanent magnets placed at the ends and a pickup coil wounded around the beam, as illustrated in Fig. 5. The excitation of the clamped end of the rod with the external vibration makes the rod bend and experience strain

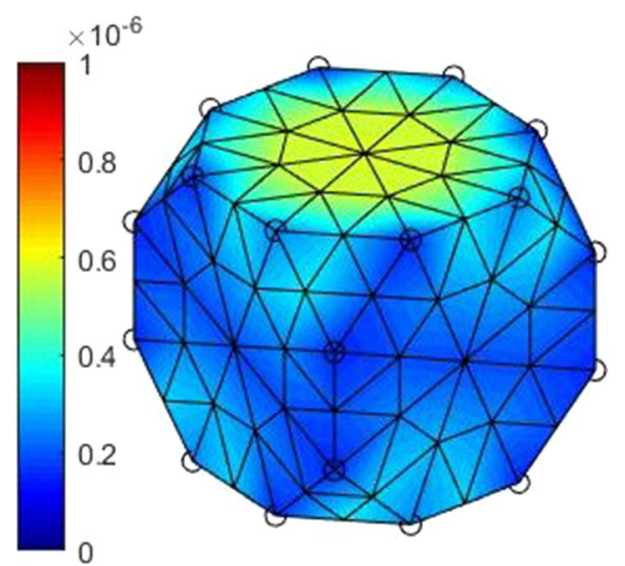

Figure 1 The magnetostrictive strain, $\lambda_{s}$, of Galfenol along the $\langle 100\rangle$ direction at $\mathrm{H}=45$ Oe.

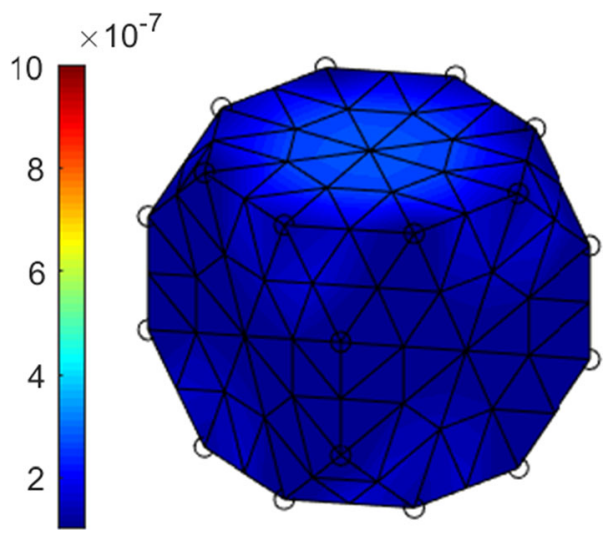

Figure 2 The magnetostrictive strain, $\lambda_{s}$, of Alfenol along the $\langle 100\rangle$ direction at $\mathrm{H}=45 \mathrm{Oe}$.

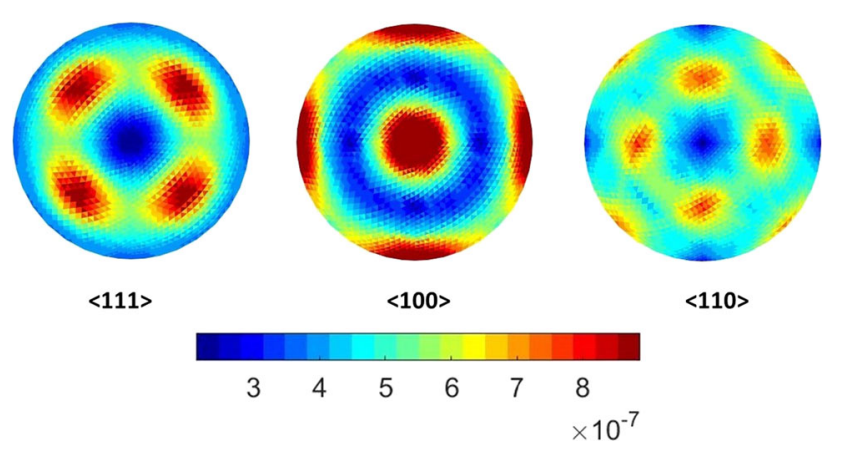

Figure 3 The pole figures in $\langle 111\rangle,\langle 100\rangle$, and $\langle 110\rangle$ directions for the magnetostrictive strain, $\lambda_{s}$, of Galfenol.

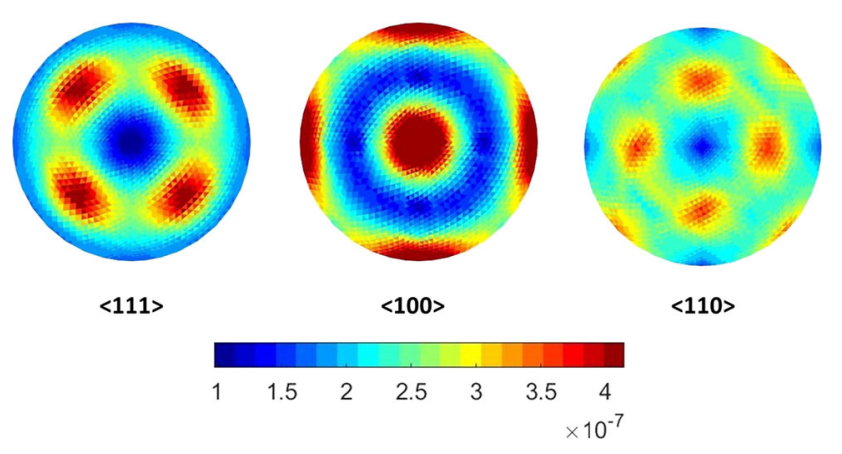

Figure 4 The pole figures in $\langle 111\rangle,\langle 100\rangle$, and $\langle 110\rangle$ directions for the magnetostrictive strain, $\lambda_{s}$, of Alfenol.

that leads to a change in the magnetic permeability of the rod. This alters the magnetic field as well as the magnetic flux for the pickup coil. From Faraday's Law, a current passes through the coil to induce the change in the magnetic flux. The induced magnetic field can be obtained through the following set of equations [49]: 
Figure 5 The schematic of the setup.

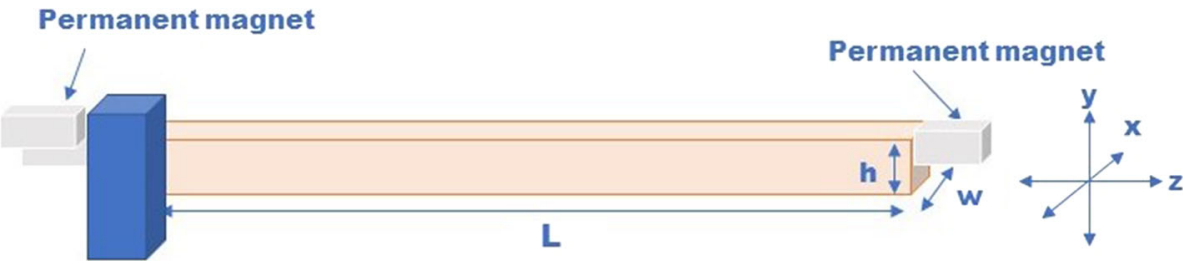

$B=\mathrm{d} \sigma+\mu . H$

Table 1 Model parameters

\begin{tabular}{lll}
\hline Parameters & Galfenol $[43,47]$ & Alfenol [50, 51] \\
\hline$\rho_{g}$ & $7800 \mathrm{~kg} / \mathrm{m}^{3}$ & $7109.7 \mathrm{~kg} / \mathrm{m}^{3}$ \\
$w$ & $0.0155 \mathrm{~m}$ & $0.0155 \mathrm{~m}$ \\
$L$ & $0.085 \mathrm{~m}$ & $0.0988 \mathrm{~m}$ \\
$h$ & $0.0075 \mathrm{~m}$ & $0.0076 \mathrm{~m}$ \\
$t_{g}$ & $0.00687 \mathrm{~m}$ & $0.00687 \mathrm{~m}$ \\
$H$ & $45 \mathrm{Oe}$ & $45 \mathrm{Oe}$ \\
$\omega_{b}$ & $2 \pi * 60 \mathrm{~s}^{-1}$ & $2 \pi * 60 \mathrm{~s}^{-1}$ \\
$\chi$ & 0.04 & 0.04 \\
$a_{0}$ & $0.2^{*} 9.81 \mathrm{~m} / \mathrm{s}^{2}$ & $0.2^{*} 9.81 \mathrm{~m} / \mathrm{s}^{2}$ \\
$K_{1}$ & $3.6 \times 10^{4} \mathrm{~J} / \mathrm{m}^{3}$ & $3.1 \times 10^{4} \mathrm{~J} / \mathrm{m}^{3}$ \\
$K_{2}$ & 0 & 0 \\
$\lambda_{100}$ & $170 \times 10^{-6}$ & $80 \times 10^{-6}$ \\
$\lambda_{111}$ & $(-14 / 3) \times 10^{-6}$ & $-3.8675 \times 10^{-6}$ \\
$R_{c}$ & $24.3 \Omega$ & $24.3 \Omega$ \\
\hline
\end{tabular}

length of the beam, $\rho$ is the mass per unit length of the beam, $I$ is the second moment of area of the beam, and $m$ is the mass of the tip load. The following relations are used to compute $I$ and $\rho$ :

$I=\frac{1}{12} w h^{3}, \quad \rho=\rho_{g} t_{g} w$

where $w$ is the width of the beam, $h$ is the total thickness of the beam, $\rho_{g}$ is the density of the material (Galfenol or Alfenol), $t_{g}$ is the thickness of the material layer (Galfenol or Alfenol). All values of the given parameters are shown in Table 1.

The input vibration is provided in sinusoidal form [43]:

$y_{b}=Y_{b} \sin \left(\omega_{b} t\right)=\frac{-a_{0}}{\omega_{b}^{2}} \sin \left(\omega_{b} t\right)$

where $Y_{b}$ corresponds to the maximum displacement, $a_{0}$ is the acceleration, and $\omega_{b}$ is the angular velocity of the vibration.

Next, the displacement of the tip mass with respect to the clamped end can be expressed with the following equation [43]:

$$
\begin{aligned}
y_{L}= & Y_{b} \sin \left(\omega_{b} t\right) \\
& -Y_{b} \omega_{n} \sqrt{\frac{\omega_{n}^{2}+4 \chi^{2} \omega_{b}^{2}}{\left(\omega_{n}^{2}-\omega_{b}^{2}\right)^{2}+4 \chi^{2} \omega_{b}^{2} \omega_{n}^{2}}} \cos \left(\omega_{b} t-\phi\right)
\end{aligned}
$$

where $\chi$ is the damping ratio, and $\phi$ is the phase difference defined as [43]:

$\phi=\arctan \left(\frac{2 \chi \omega_{n} \omega_{b}}{\omega_{n}^{2}-\omega_{b}^{2}}\right)+\arctan \left(\frac{\omega_{n}}{2 \chi \omega_{b}}\right)$

The strain emerging due to this vibration can be written in the following way [43]:

$\epsilon=\frac{3(x-L) z}{L^{3}} y_{L}(t)$

where $x$ and $z$ are the coordinates along the length and thickness directions, respectively.

The magnetic flux passing through the cross section area of the beam, $A_{\text {beam }}$, is as follows:

$\Phi=\oint B \cdot \mathrm{d} A_{\text {beam }}$ 
From Faraday's Law, the change in the flux creates a voltage across the coil wounded around the beam. Moreover, combining Eq. (16) with Eq. (17), enables the induced magnetic field to be expressed in terms of the strain and coupling coefficient.

After combining the induced magnetic field expression with Faraday's Law and integrating it along the length of the beam, the following expression is attained:

$V(t)=-\frac{\mathrm{d} \Phi}{\mathrm{d} t}$

$V(t)=-n \frac{w}{L} d^{*} E \frac{\mathrm{d} y_{L}}{\mathrm{~d} t} \int_{-\frac{h}{2}}^{t_{g}-\frac{h}{2}} \int_{0}^{L} \frac{3(x-L) z}{L^{3}} \mathrm{~d} x \mathrm{~d} z$

To find the produced voltage, the root mean square value of the voltage function is evaluated as follows:

$V_{\text {rms }}=\sqrt{\frac{w_{b}}{2 \pi} \int_{0}^{2 \pi / w_{b}}(V(t))^{2} \mathrm{~d} t}$

For the maximum power output, the resistor connected to the coil should be equal to the resistance of the coil. Therefore, the voltage across the connected resistor is equal to the half of the RMS voltage produced. The output power can be written in terms of the RMS value of the voltage:
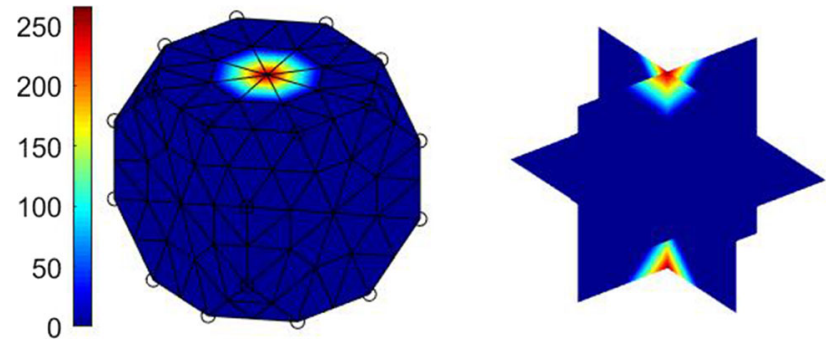

Figure 6 The optimum ODF representation of the optimum Galfenol microstructure for maximum efficiency $(\eta=80.47 \%)$.
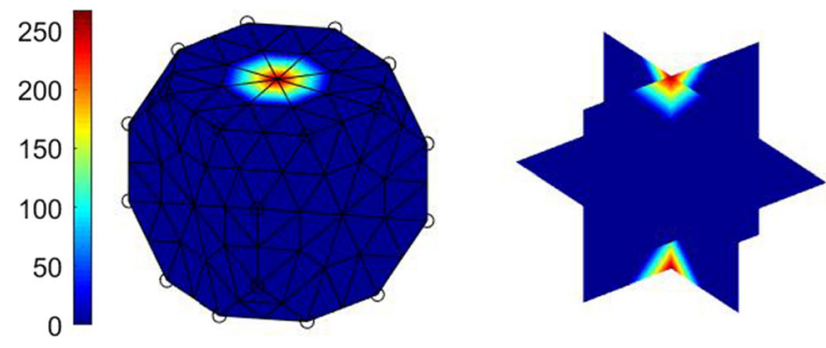

Figure 7 The optimum ODF representation of the optimum Alfenol microstructure for maximum efficiency $(\eta=32.36 \%)$.
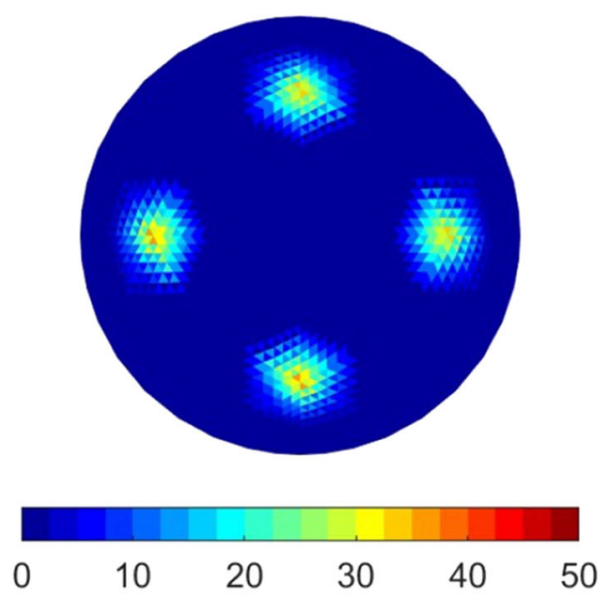

\section{$<111>$}
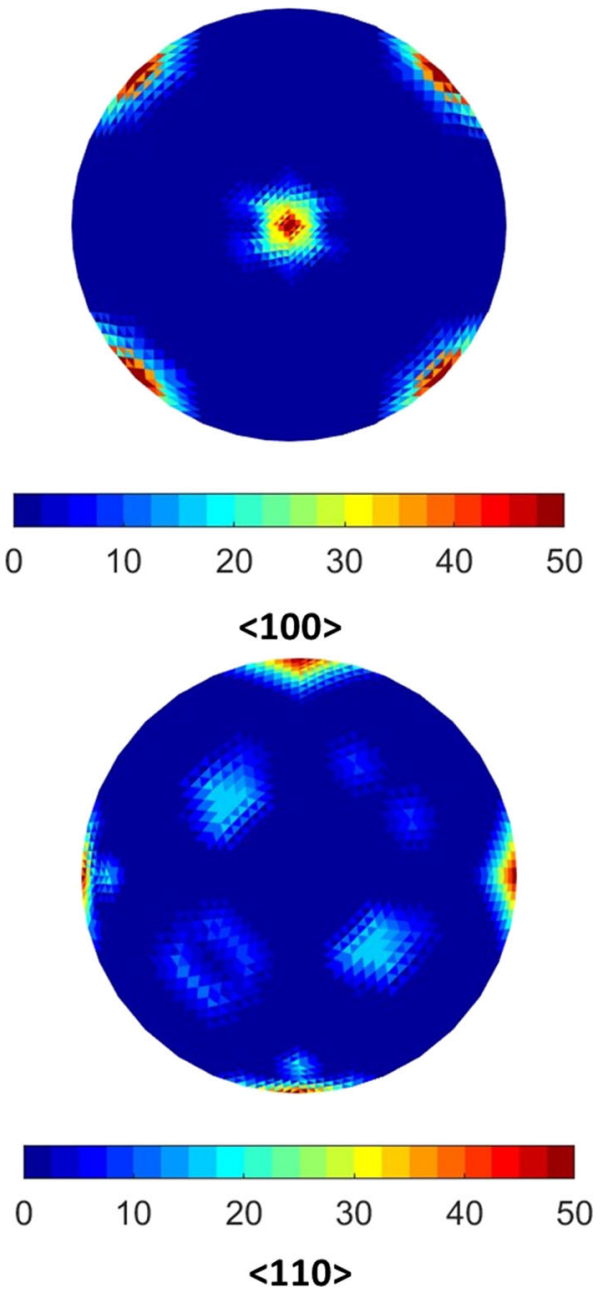

Figure $8 \mathrm{PFs}$ for optimum microstructure of Galfenol for maximum efficiency. 

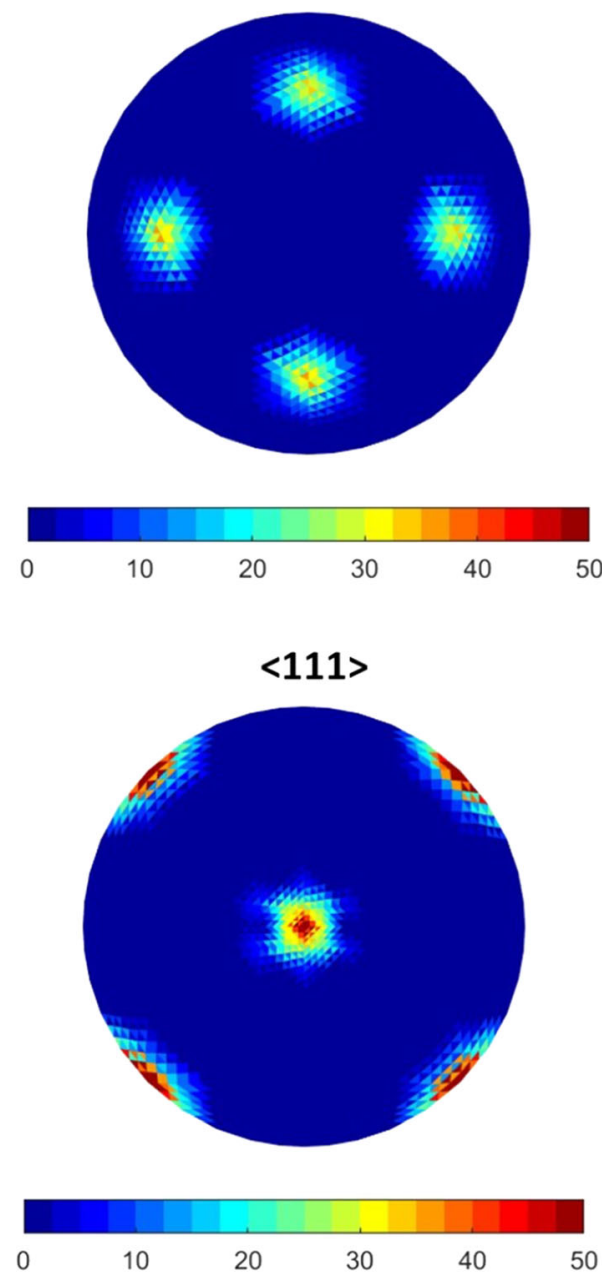

$<100>$

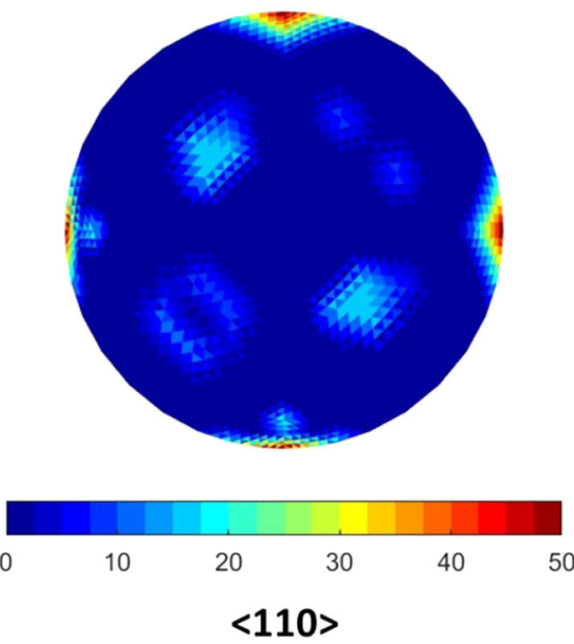

Figure 9 PFs for optimum microstructure of Alfenol for maximum efficiency.
$P_{\text {out-rms }}=\frac{V_{\mathrm{rms}}^{2}}{4 R_{c}}$

where $R_{c}$ is the resistance of the coil, $V_{\text {rms }}$ is the rms voltage evaluated in Eq. (27).

The energy produced by the input force due to the vibration can be expressed using the following equation:

$E(t)=F(t) y_{b}(t)=(0.2235 \rho L+m) \frac{\mathrm{d}^{2} y_{b}}{\mathrm{~d} t^{2}} y_{b}$

which can also be written as:

$E(t)=(0.2235 \rho L+m) a_{0} Y_{b} \sin ^{2}\left(\omega_{b} t\right)$

The derivative of the work done with respect to time gives the power as a function of time as follows:

$P_{\text {in }}(t)=\frac{\mathrm{d} E}{\mathrm{~d} t}=(0.2235 \rho L+m) a_{0} Y_{b} \omega_{b} \sin \left(2 \omega_{b} t\right)$

Next, the root mean square of the input power can be determined in a similar way:

$P_{\text {in }-\mathrm{rms}}=\sqrt{\frac{w_{b}}{2 \pi} \int_{0}^{2 \pi / w_{b}}\left(P_{\text {in }}(t)\right)^{2} \mathrm{~d} t}$

The energy efficiency is calculated as follows:

$\eta=\frac{P_{\text {out }- \text { rms }}}{P_{\text {in-rms }}}$

\section{Optimization of Galfenol and Alfenol microstructure}

The aim of the optimization problem is to find the optimum microstructure design giving the highest efficiency for the energy harvesting mechanism. The optimization is implemented using the Sequential
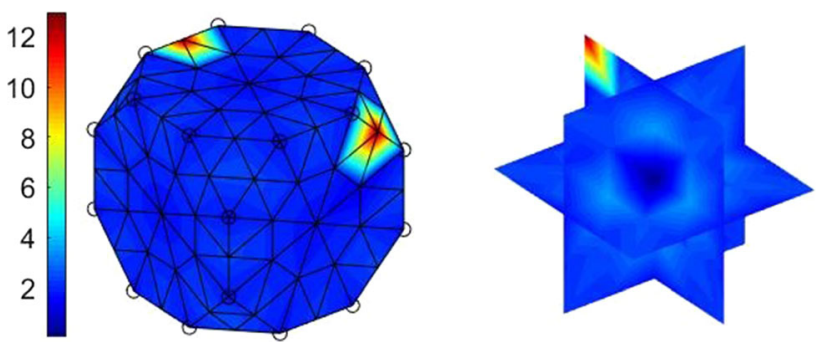

Figure 10 The optimum ODF representation of the optimum Galfenol microstructure for maximum Young's modulus $(E=134.8104 \mathrm{GPa})$. 

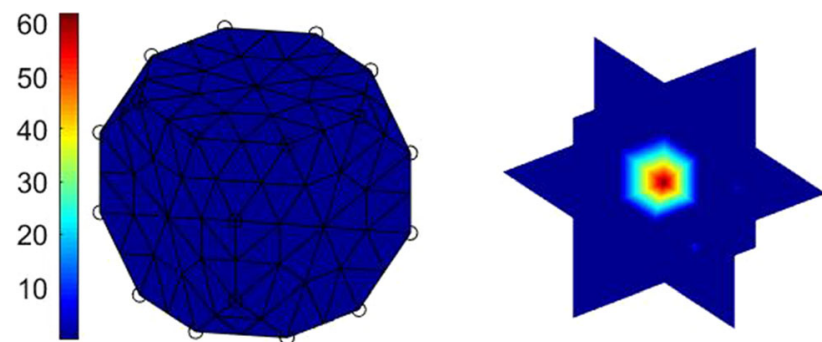

Figure 11 The optimum ODF representation of the optimum Alfenol microstructure for maximum Young's modulus $(E=130.1275 \mathrm{GPa})$.

Quadratic Programming (SQP) algorithm by inputting the randomly oriented texture sample as the initial guess. We considered all crystallographic orientations (76 unique orientations we model through the ODFs) as design variables in the optimization problem. The mathematical formulation of the optimization problem is as follows:

Figure 12 Pole figures of Galfenol in $\langle 111\rangle,\langle 100\rangle$, and $\langle 110\rangle$ directions for maximum Young's modulus.
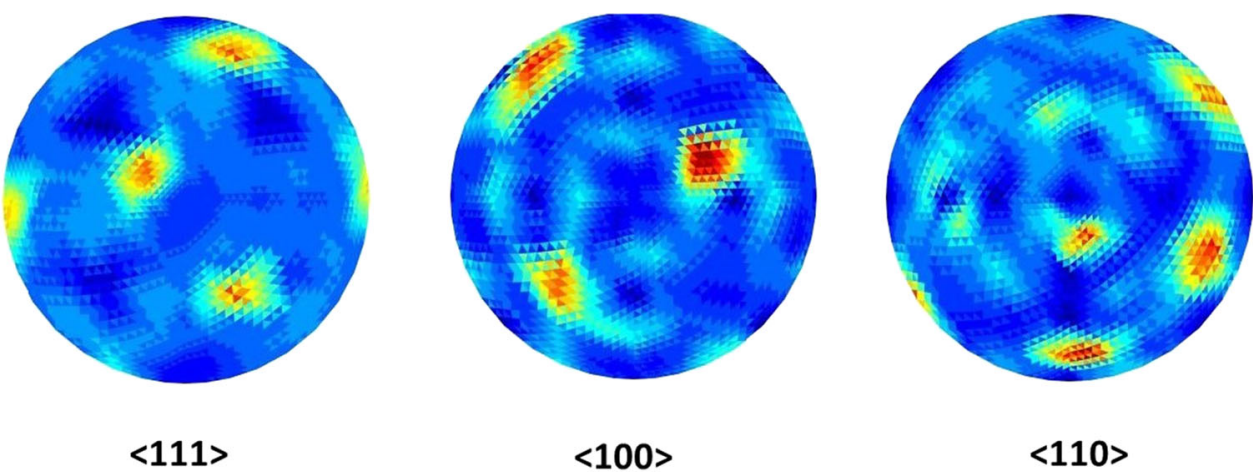

$<110>$
Figure 13 Pole figures of Alfenol in $\langle 111\rangle,\langle 100\rangle$, and $\langle 110\rangle$ directions for maximum Young's modulus.
$\operatorname{Max} \eta$

Subject to $q^{T} A=1$

$A \geq 0$

The optimum microstructure design for this problem is found to produce an $\eta$ (efficiency of energy harvesting) value of $80.47 \%$ for Galfenol and $32.36 \%$ for Alfenol. However, when compared to the output power of the baseline randomly oriented microstructural texture, the optimized Galfenol microstructure improves the output power about 111 times, whereas the optimum Alfenol provides the 61 times of the baseline value. Therefore, a much more significant enhancement is achieved by optimizing the microstructural texture of Galfenol. The efficiency of the optimum microstructure designs is also higher than the observed efficiency values in commercial products of the same materials.
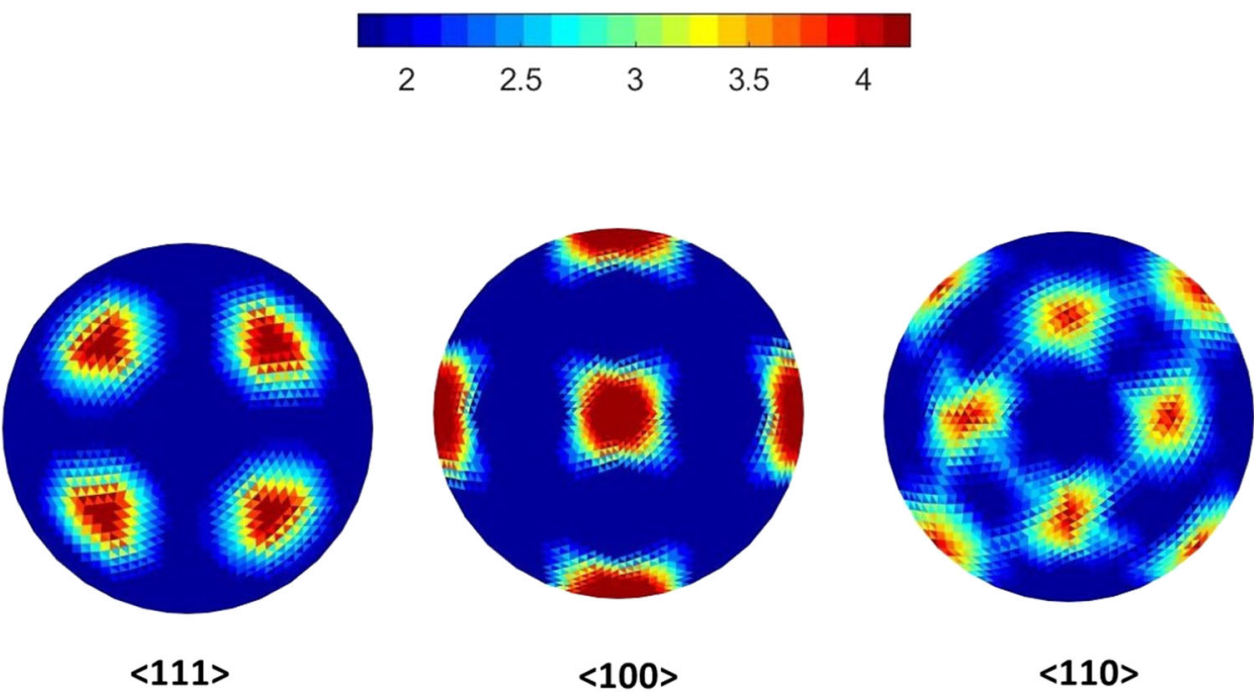

$<110>$ 
The ODFs of the optimum Galfenol and Alfenol microstructures are visualized in Figs. 6 and 7, respectively. The pole figures of the optimum microstructures are provided in Figs. 8 and 9 for Galfenol and Alfenol (in the $\langle 111\rangle,\langle 100\rangle$, and $\langle 110\rangle$ directions), respectively.

As deduced from Figs. 6, 7, 8 and 9, the optimum textures of Galfenol and Alfenol for the highest energy efficiency are very similar. This result indicates that the magneto-mechanical coupling in
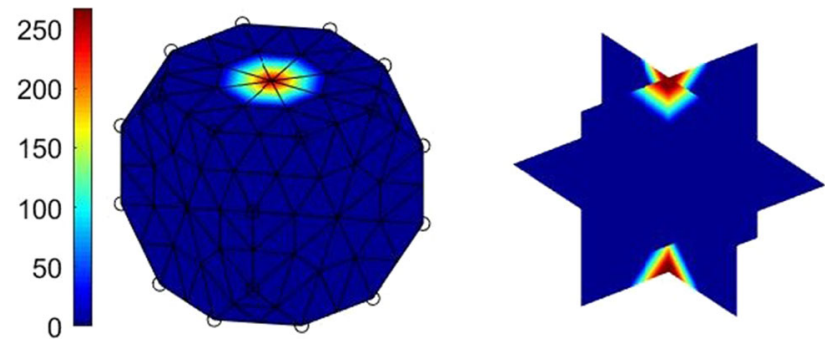

Figure 14 The optimum ODF representation of the optimum Galfenol microstructure for maximum average magnetostrictive strain $\left(\lambda_{s}=156.13 \times 10^{-6}\right)$.
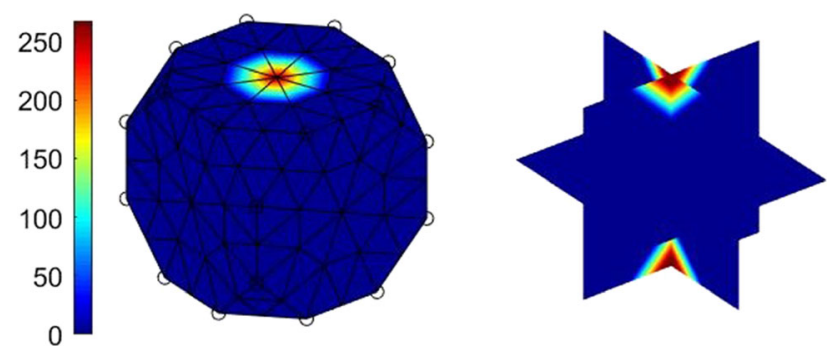

Figure 15 The optimum ODF representation of the optimum Alfenol microstructure for maximum average magnetostrictive strain $\left(\lambda_{s}=73.131 \times 10^{-6}\right)$.

Figure 16 Pole figures of Galfenol in $\langle 111\rangle,\langle 100\rangle$, and $\langle 110\rangle$ directions for maximum average magnetostrictive strain. magnetostrictive materials can be improved by similar microstructural texture components even though the higher magnetization directions are different.

Then, the ODFs providing highest Young's modulus value for Galfenol and Alfenol can be investigated as follows.

$\operatorname{Max} E$

Subject to $q^{T} A=1$

$A \geq 0$

The ODFs of optimum Galfenol and Alfenol microstructures for maximum Young's modulus are visualized in Figs. 10 and 11. The pole figures of these optimum microstructures are given in Figs. 12 and 13 for Galfenol and Alfenol, respectively.

Then, the ODFs providing highest average magnetostrictive strain value for Galfenol and Alfenol can be investigated as follows.

$\operatorname{Max} \lambda_{s}$

Subject to $q^{T} A=1$

$A \geq 0$

The ODFs of optimum Galfenol and Alfenol microstructures for maximum average magnetostrictive strain are visualized in Figs. 14 and 15. The pole figures of these optimum microstructures are given in Figs. 16 and 17 for Galfenol and Alfenol, respectively. Energy efficiency, Young's modulus and average magnetostrictive values are calculated for optimum ODFs of Galfenol and Alfenol to achieve highest efficiency (Solution 1), highest average magnetostrictive strain (Solution 2)

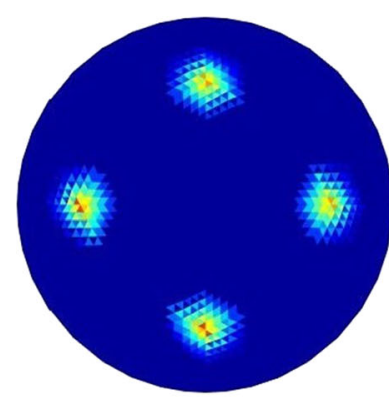

$<111>$

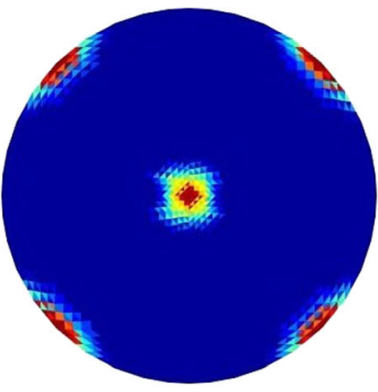

$<100>$

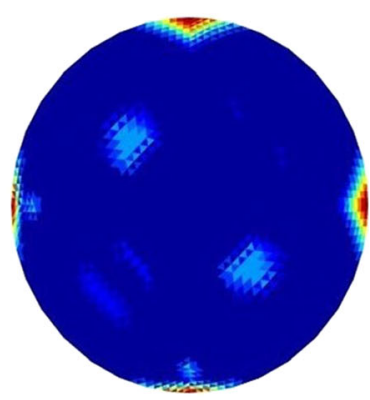

$<110>$ 
Figure 17 Pole figures of Alfenol in $\langle 111\rangle,\langle 100\rangle$, and $\langle 110\rangle$ directions for maximum average magnetostrictive strain.
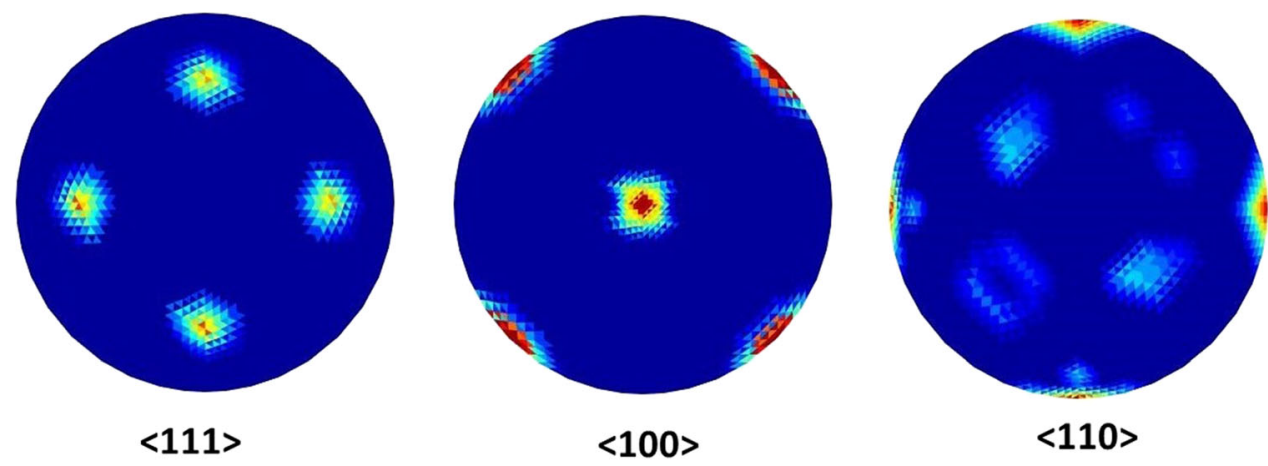

10

30

40

Table 2 Values for Galfenol

\begin{tabular}{lccc}
\hline Design & $\eta(\%)$ & $E(\mathrm{GPa})$ & $\lambda_{s} \times 10^{-6}$ \\
\hline Solution 1 & 80.47 & 72.5993 & 155.63 \\
Solution 2 & 3.8 & 71.9468 & 156.13 \\
Solution 3 & 0.44 & 134.8104 & 83.899 \\
\hline
\end{tabular}

Table 3 Values for Alfenol

\begin{tabular}{lrcc}
\hline Design & $\eta(\%)$ & $E(\mathrm{GPa})$ & $\lambda_{s} \times 10^{-6}$ \\
\hline Solution 1 & 32.36 & 114.0656 & 73.131 \\
Solution 2 & 32.36 & 114.0656 & 73.131 \\
Solution 3 & 6.72 & 130.1275 & 66.186 \\
\hline
\end{tabular}

and highest Young's modulus value (Solution 3), as listed in Tables 2 and 3.

\section{Conclusion}

The present work addresses a computational study to design magnetostrictive Galfenol and Alfenol microstructures for bending mode energy harvesters. Galfenol and Alfenol have been appealed much interest in energy harvesting due to their bio-directional coupling properties. To improve the efficiency of the energy harvesters, we perform a design optimization to find the optimum microstructural textures. Our results indicate an energy efficiency improvement over $80 \%$, which is higher than the observed efficiency increases in commercial products of the same materials. The future work may focus on the efforts towards manufacturing these optimum microstructure designs using conventional processing techniques or 3-D printing.

\section{Funding}

We acknowledge the faculty start-up package provided by the Mechanical Engineering Department at Virginia Polytechnic Institute and State University.

\section{Compliance with ethical standards}

Conflict of interest The authors declare that they have no conflict of interest.

\section{References} materials. Academic Press, San Diego

[2] Saito H (1961) Effect of aging on the maximum permeability in quenched $\mathrm{Fe}-\mathrm{Al}$ alloys (ALPERM). Trans Jpn Inst Met 2:187. https://doi.org/10.2320/matertrans1960.2.187

[3] Restorff JB, Wun-Fogle M, Hathaway KB, Clark AE, Lograsso TA, Petculescu G (2012) Tetragonal magnetostriction and magnetoelastic coupling in $\mathrm{Fe}-\mathrm{Al}, \mathrm{Fe}-\mathrm{Ga}, \mathrm{Fe}-\mathrm{Ge}, \mathrm{Fe}-$ $\mathrm{Si}$, Fe-Ga-Al, and $\mathrm{Fe}-\mathrm{Ga}-\mathrm{Ge}$ alloys. J Appl Phys 111:023905. https://doi.org/10.1063/1.3674318

[4] Pasnak M, Lundsten R (1959) Effects of high temperature on magnetic properties of core materials. J Appl Phys 30:107-108. https://doi.org/10.1063/1.2185844

[5] Lufcy CW (1959) Magnetic transducer head for tape recording, playback and erasing. https://patents.google.com/ patent/US2912515A/en. Accessed 14 Mar 2020
[1] Engdahl G (2000) Handbook of giant magnetostrictive 
[6] Na S, Rice M, Raghunath G, Klimchenko V, Flatau A (2014) Magnetostrictive Alfenol Whisker sensor performance and sensitivity to Whisker thickness. IEEE Trans Magn 50:1-4. h ttps://doi.org/10.1109/TMAG.2014.2324413

[7] Raghunath G, Flatau AB, Na S, Barkley B (2014) Development of a bio-inspired tactile magnetostrictive whisker sensor using alfenol. In: Proceedings of the ASME 2014 conference on smart materials, adaptive structures and intelligent systems. https://doi.org/10.1115/smasis2014-7550

[8] Muller B, Van Order MN, Na SM, Flatau AB (2019) Alfenol patch-, galfenol patch- and galfenol paint-based torque sensor characterization studies. AIP Adv 9:045113. https://doi. org/10.1063/1.5080140

[9] Clark AE, Restorff JB, Wun-Fogle M, Lograsso TA, Schlagel DL (2000) Magnetostrictive properties of body-centered cubic $\mathrm{Fe}-\mathrm{Ga}$ and $\mathrm{Fe}-\mathrm{Ga}-\mathrm{Al}$ alloys. IEEE Trans Magn 36:3238-3240. https://doi.org/10.1109/20.908752

[10] Summers E, Lograsso T, Wun-Fogle M (2007) Magnetostriction of binary and ternary Fe-Ga alloys. J Mater Sci 42:9582-9594. https://doi.org/10.1007/s10853-007-2096-6

[11] Clark AE, Wun-Fogle Restorff JB, Lograsso TA (2002) Magnetostrictive properties of Galfenol alloys under compressive stress. Mater Trans 43:881-886. https://doi.org/10. 2320/matertrans. 43.881

[12] Jen SU, Lo YY, Pai LW (2016) Temperature dependence of mechanical properties of the $F e_{81} G a_{19}$ (Galfenol) alloy. J Phys D 49:145004. https://doi.org/10.1088/0022-3727/49/ 14/145004

[13] Evans P, Dapino M (2011) Dynamic model for 3-D magnetostrictive transducers. IEEE Trans Magn 47:221-230. h ttps://doi.org/10.1109/tmag.2010.2088130

[14] Graham FC, Mudivarthi C, Datta S, Flatau AB (2009) Modeling of a Galfenol transducer using the bidirectionally coupled magnetoelastic model. Smart Mater Struct 18:104013. https://doi.org/10.1088/0964-1726/18/10/ 104013

[15] Kellogg RA, Flatau AB, Clark AE, Wun-Fogle M, Lograsso $\mathrm{T}$ (2005) Quasi-static transduction characterization of Galfenol. J Intell Mater Syst Struct 16:471-479. https://doi.org/ 10.1177/1045389x05050107

[16] Basantkumar RR, Stadler BJ, Robbins WP, Summers E (2006) Integration of thin-film Galfenol with MEMS cantilevers for magnetic actuation. IEEE Trans Magn 42:3102-3104. https://doi.org/10.1109/tmag.2006.879666

[17] Shu L, Wu G, Chen D, Dapino MJ (2016) Modeling of galfenol bending actuator considering nonlinear hysteresis and dynamic real-time control strategy. Smart Mater Struct 25:035046. https://doi.org/10.1088/0964-1726/25/3/035046

[18] Ghodsi M, Modabberifar M, Ueno T (2011) Quality factor, static and dynamic responses of miniature galfenol actuator at wide range of temperature. Int J Phys Sci 6:8143-8150. h ttps://doi.org/10.5897/ijps11.918

[19] Datta S, Atulasimha J, Flatau AB (2009) Figures of merit of magnetostrictive single crystal irongallium alloys for actuator and sensor applications. J Magn Magn Mater 321:4017-4031. https://doi.org/10.1016/j.jmmm.2009.07. 067

[20] Datta S, Atulasimha J, Flatau AB (2007) Modeling of magnetostrictive Galfenol sensor and validation using four point bending test. J Appl Phys 101:09C521. https://doi.org/ 10.1063/1.2714306

[21] Downey PR, Flatau AB (2005) Magnetoelastic bending of Galfenol for sensor applications. J Appl Phys 97:10R505. h ttps://doi.org/10.1063/1.1853838

[22] Yoo J, Marschner U, Flatau AB (2005) Preliminary Galfenol vibratory gyro-sensor design. Proc SPIE 5764:111-119. h ttps://doi.org/10.1117/12.599973

[23] Sauer S, Marschner U, Adolphi B, Clasbrummel B, Fischer W (2012) Passive wireless resonant Galfenol sensor for osteosynthesis plate bending measurement. IEEE Sens J 12:1226-1233. https://doi.org/10.1109/jsen.2011.2167747

[24] Nair B, Nachlas JA, Murphree Z (2014) Magnetostrictive devices and systems. https://patents.google.com/patent/U S9438138B2/en. Accessed 14 Mar 2020

[25] Murphree Z (2013) Magnetostrictive wave energy harvester with heave plate. https://patents.google.com/patent/W O2014004699A1/tr. Accessed 14 Mar 2020

[26] Dudley JH, Nachlas JA (2014) Vibration energy harvester. h ttps://patents.google.com/patent/US20140284937A1/en. Accessed 14 Mar 2020

[27] Liu H, Wang S, Zhang Y, Wang W (2014) Study on the giant magnetostrictive vibration-power generation method for battery-less tire pressure monitoring system. Proc Inst Mech Eng C-J MEC 229:1639-1651. https://doi.org/10.1177/ 0954406214545821

[28] Park Y, Kang H, Wereley N (2014) Conceptual design of rotary magnetostrictive energy harvester. J Appl Phys 115:17E713. https://doi.org/10.1063/1.4865976

[29] Berbyuk V (2013) Vibration energy harvesting using Galfenol-based transducer. Proc SPIE 8688:86881F. https://doi. org/10.1117/12.2009812

[30] Staley ME, Flatau AB (2005) Characterization of energy harvesting potential of Terfenol-D and Galfenol. Proc SPIE 5764:630-640. https://doi.org/10.1117/12.604871

[31] Ueno T, Yamada S (2011) Performance of energy harvester using irongallium alloy in free vibration. IEEE Trans Magn 47:2407-2409. https://doi.org/10.1109/tmag.2011.2158303

[32] Kita S, Ueno T, Yamada S (2015) Improvement of force factor of magnetostrictive vibration power generator for high 
efficiency. J Appl Phys 117:17B508. https://doi.org/10.1063/ 1.4907237

[33] Ueno T (2015) Performance of improved magnetostrictive vibrational power generator, simple and high power output for practical applications. J Appl Phys 117:17A740. https://d oi.org/10.1063/1.4917464

[34] Deng Z, Dapino M (2016) Influence of electrical impedance and mechanical bistability on Galfenol-based unimorph harvesters. J Intell Mater Syst Struct 28:421-431. https://doi. org/10.1177/1045389x16666176

[35] Park J, Na S, Raghunath G, Flatau A (2016) Stress-annealinduced magnetic anisotropy in highly textured $\mathrm{Fe}-\mathrm{Ga}$ and $\mathrm{Fe}-\mathrm{Al}$ magnetostrictive strips for bending-mode vibrational energy harvesters. AIP Adv 6:056221. https://doi.org/10.10 63/1.4944772

[36] Park J, Na S, Flatau A (2017) Field-anneal-induced magnetic anisotropy in highly textured $\mathrm{Fe}-\mathrm{Al}$ magnetostrictive strips. AIP Adv 7:056431. https://doi.org/10.1063/1.4978006

[37] Allison J, Backman D, Christodoulou L (2006) Integrated computational materials engineering: a new paradigm for the global materials profession. JOM 58:25-27. https://doi.org/ 10.1007/s11837-006-0223-5

[38] Ashby M (1992) Materials selection in mechanical design, 1st edn. Pergamon Press, New York

[39] Olson G (1997) Computational design of hierarchically structured materials. Science 277:1237-1242. https://doi.org/ 10.1126/science. 277.5330 .1237

[40] Acar P, Sundararaghavan V (2016) Linear solution scheme for microstructure design with process constraints. AIAA J 54:4022-4031. https://doi.org/10.2514/1.j055247

[41] Acar P, Srivastava S, Sundararaghavan V (2017) Stochastic design optimization of microstructures with utilization of a linear solver. AIAA J 55:3161-3168. https://doi.org/10.251 4/1.j056000

[42] Acar P, Sundararaghavan V (2019) Stochastic design optimization of microstructural features using linear programming for robust material design. AIAA J 57:448-455. h ttps://doi.org/10.2514/1.j057377
[43] Haynes RA, Yoo J, Flatau AB (2013) Performance of a bending mode energy harvester using $\mathrm{Fe}-\mathrm{Ga}$ alloy (Galfenol). Proc SPIE 8728:87280U. https://doi.org/10.1117/12. 2016424

[44] Acar P, Sundararaghavan V (2016) Utilization of a linear solver for multiscale design and optimization of microstructures. AIAA J 54:1751-1759. https://doi.org/10. 2514/1.j054822

[45] Acar P, Sundararaghavan V (2017) Uncertainty quantification of microstructural properties due to variability in measured pole figures. Acta Mater 124:100-108. https://doi.org/ 10.1016/j.actamat.2016.10.070

[46] Acar P, Sundararaghavan V (2017) Uncertainty quantification of microstructural properties due to experimental variations. AIAA J 55:2824-2832. https://doi.org/10.2514/1. j055689

[47] Kumar A, Sundararaghavan V (2017) Simulation of magnetostrictive properties of Galfenol under thermomechanical deformation. Finite Elem Anal Des 127:1-5. https://doi.org/ 10.1016/j.finel.2016.11.009

[48] Dapino MJ, Smith RC, Faidley LE, Flatau AB (2000) A coupled structural-magnetic strain and stress model for magnetostrictive transducers. J Intell Mater Syst Struct 11:135-152. https://doi.org/10.1177/104538900772664332

[49] Chopra I, Sirohi J (2014) Smart structures theory, 1st edn. Cambridge University Press, New York

[50] Leamy HJ, Gibson ED, Kayser FX (1967) The elastic stiffness coefficients of iron-aluminum alloys- experimental results and thermodynamic analysis. Acta Metall 15:1827-1838. https://doi.org/10.1016/0001-6160(67)90047 $-8$

[51] Zhang Y, Wang H, Wu R (2012) First-principles determination of the rhombohedral magnetostriction of $F e_{100 x} A l_{x}$ and $F e_{100 x} G a_{x}$ alloys. Phys Rev B 86:224410. https://doi. org/10.1103/PhysRevB.86.224410

Publisher's Note Springer Nature remains neutral with regard to jurisdictional claims in published maps and institutional affiliations. 